TRANSACTIONS OF THE

AMERICAN MATHEMATICAL SOCIETY

Volume 364, Number 7, July 2012, Pages 3685-3698

S 0002-9947(2012)05532-2

Article electronically published on February 16, 2012

\title{
A FINITENESS RESULT FOR COMMUTING SQUARES WITH LARGE SECOND RELATIVE COMMUTANT
}

\author{
REMUS NICOARA
}

\begin{abstract}
We prove that there exist only finitely many commuting squares of finite dimensional $*$-algebras of fixed dimension, satisfying a "large second relative commutant" condition. We show this by studying the local minima of $w \rightarrow \operatorname{dim}\left(A \cap w B w^{*}\right)$, where $A, B$ are fixed subalgebras of some *-algebra $C$ and $w \in C$ is a unitary.

When applied to lattices arising from subfactors satisfying a certain extremality-like condition, our result yields Ocneanu's finiteness theorem for the standard invariants of such finite depth subfactors.
\end{abstract}

\section{INTRODUCTION}

Commuting squares were introduced by S. Popa in Po1] (see also [Po2, [JS]). They arise naturally in subfactor theory, as invariants and construction data for subfactors. A commuting square is a square of inclusions of finite dimensional *-algebras:

$$
\mathfrak{C}=\left(\begin{array}{ccc}
P_{-1} & \subset & P_{0} \\
\cup & & \cup, \tau \\
Q_{-1} & \subset & Q_{0}
\end{array}\right)
$$

with a faithful trace $\tau$ on $P_{0}$, such that

$$
P_{-1} \ominus Q_{-1} \perp Q_{0} \ominus Q_{-1},
$$

i.e. the vector spaces $P_{-1} \ominus Q_{-1}$ and $Q_{0} \ominus Q_{-1}$ are orthogonal with respect to the inner product defined by $\tau$ on $P_{0}$.

In this paper we consider commuting squares that satisfy a "large second relative commutant" (LRC) condition. These are commuting squares with a $\lambda$-Markov trace $\tau$ such that, after doing Jones' basic construction ([Jon] :

$$
\mathfrak{L}=\left(\begin{array}{ccccc}
P_{-1} & \subset & P_{0} & \stackrel{e}{\subset} & P_{1} \\
\cup & & \cup & & \cup \\
Q_{-1} & \subset & Q_{0} & \stackrel{e}{\subset} & Q_{1}
\end{array}\right),
$$

the relative commutant $R_{1}=P_{-1}^{\prime} \cap Q_{1}$ satisfies

$$
E_{P_{0}}\left(R_{1}^{\prime} \cap P_{1}\right)=P_{-1},
$$

where $E_{P_{0}}$ denotes the projection from the vector space $P_{1}$ onto $P_{0}$, with respect to the inner product defined by $\tau$. Since in general $P_{-1}=E_{P_{0}}\left(P_{-1}\right) \subset E_{P_{0}}\left(R_{1}^{\prime} \cap P_{1}\right)$, we can interpret the equality as a restriction on $R_{1}^{\prime}$ and thus a largeness condition for $R_{1}$.

Received by the editors August 19, 2010 and, in revised form, December 26, 2010.

2010 Mathematics Subject Classification. Primary 46L37.

(C)2012 American Mathematical Society Reverts to public domain 28 years from publication 
We prove that there exist only finitely many such LRC commuting squares with $\operatorname{dim}\left(P_{0}\right)$ fixed (Theorem 2.4).

The proof is based on derivation techniques similar to those we introduced in [Ni]. We also need some properties of the local minima of maps $w \rightarrow \operatorname{dim}\left(A \cap w B w^{*}\right)$, where $A, B$ are fixed subalgebras of some *-algebra $C$. More precisely, we show that if $\operatorname{dim}(A \cap B) \leq \operatorname{dim}\left(A \cap w_{n} B w_{n}^{*}\right)$ for some unitaries $w_{n} \rightarrow I$ in $C$, then any direction of convergence of $w_{n}$ (in the sense of Definition 2.8) belongs to $A+B+(A \cap B)^{\prime} \cap C$ (Proposition 3.3.).

The finiteness result of this paper, as well as the LRC condition, are motivated by the case of commuting squares arising in the standard invariant of a subfactor.

Let us recall the definition of the standard invariant. Let $N \subset M$ be an inclusion of $I I_{1}$ factors of finite index with trace $\tau$, and let $N \subset M \stackrel{e_{1}}{\subset} M_{1} \stackrel{e_{2}}{\subset} M_{2} \subset \ldots$ be the tower of factors obtained by iterating Jones' basic construction (see Jon]), where $e_{1}, e_{2}, \ldots$ denote the Jones projections. The standard invariant $\mathcal{G}_{N, M}$ is then defined as the trace-preserving isomorphism class of the following sequence of commuting squares of inclusions of finite dimensional $*$-algebras:

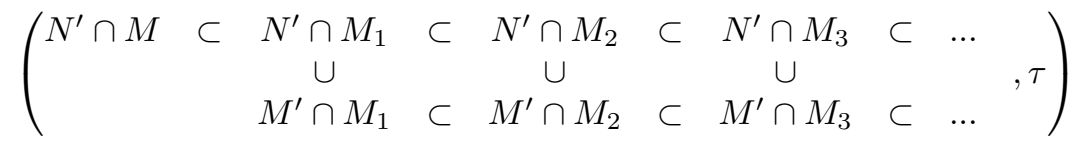

together with the Jones projections $e_{i} \in N^{\prime} \cap M_{i}$ and the trace $\tau$.

If the subfactor is of finite depth $n$, by Po1 the commuting square

$$
\mathfrak{C}_{n}=\left(\begin{array}{ccc}
N^{\prime} \cap M_{n-1} & \subset & N^{\prime} \cap M_{n} \\
\cup & & \cup \\
M^{\prime} \cap M_{n-1} & \subset & M^{\prime} \cap M_{n}
\end{array}, \tau\right)
$$

uniquely determines the subfactor. In particular, the isomorphism class of a depth 2 subfactor is uniquely determined by $\mathfrak{C}_{1}$. Moreover, if $N \subset M$ is of depth $n$, then $N \subset M_{n}$ has depth 2. This allows us to work only with depth 2 subfactors for the purpose of finiteness results.

By a seminal result of S. Popa ( $[\mathrm{Po} 2,[\mathrm{Po} 4)$, the standard invariant of a subfactor can be thought of as an abstract group-like object, described by a set of axioms. One of these axioms is based on the following equality:

$$
\left(N^{\prime} \cap M_{1}\right)^{\prime} \cap\left(M^{\prime} \cap M_{3}\right)=M_{1}^{\prime} \cap M_{3} .
$$

Thus, for the commuting square $\mathfrak{C}=\mathfrak{C}_{1}$ of a depth 2 subfactor the relative commutant $P_{-1}^{\prime} \cap Q_{1}$ is large, of dimension equal to $\operatorname{dim}\left(P_{-1}\right)$. This is our inspiration for looking for some "large relative commutant" condition.

When applied to such commuting squares $\mathfrak{C}$, the LRC condition is equivalent to the following extremality condition:

$$
E_{\left(N^{\prime} \cap M_{1}\right)^{\prime} \cap\left(N^{\prime} \cap M_{2}\right)}\left(e_{2}\right) \in \mathbb{C} I .
$$

Thus our theorem yields a finiteness result for the standard invariant of such (depth 2) subfactors. This extremality condition is not automatically true for any depth 2 subfactor, as we will show in the last section. We show however that it is true if $N^{\prime} \cap M$ is a (type $\mathrm{I}_{\mathrm{k}}$ ) factor. In particular it is true when $N^{\prime} \cap M=\mathbb{C}$, i.e. for those subfactors arising from Hopf algebras $([\mathrm{Szy}])$. It is also true for a larger class of depth 2 subfactors, those admiting an orthonormal basis for $N^{\prime} \cap M \subset N^{\prime} \cap M_{1}$ which is invariant under taking adjoints. 
We mention that this finiteness theorem for standard invariants of finite depth subfactors is well known to specialists, as a theorem of A. Ocneanu, even without the extra assumption $E_{\left(N^{\prime} \cap M_{1}\right)^{\prime} \cap\left(N^{\prime} \cap M_{2}\right)}\left(e_{2}\right) \in \mathbb{C} I$. See also EtNiOs. The proof we obtain here, as a consequence of our main theorem, is elementary in nature and does not use the languages of paragroups or tensor categories.

\section{Preliminaries}

In this section we recall the definition of a commuting square and we introduce some notation and normalizations. All the algebras considered will be matrix algebras, i.e. ${ }^{*}$-closed unital subalgebras of $\mathbb{M}_{n}(\mathbb{C})$ for some $n \geq 1$. Such an algebra is always of the form $\bigoplus_{i} \mathbb{M}_{n_{i}}(\mathbb{C})$, with $n_{i}$ positive integers.

For a unitary inclusion of matrix algebras $B \subset A$ we will use the notation:

$$
\begin{gathered}
B^{\prime} \cap A=\{\text { all } b \in B \text { such that } b a=a b \text { for all } a \in A\}, \\
\mathcal{N}_{A}(B)=\left\{u \in A \text { unitary such that } u B u^{*} \subset B\right\} .
\end{gathered}
$$

If $\tau$ is a fixed trace on $A$, we denote by $E_{B}=E_{B}^{A}$ the $\tau$-invariant conditional expectation of $A$ onto $B$.

If $B_{1}, B_{2}$ are subspaces of $A$, we denote by $E_{B_{1}}\left(B_{2}\right)$ the projection of $B_{2}$ onto $B_{1}$, with respect to the trace $\tau$. While this may be considered an abuse of notation, it is consistent with the conditional expectation notation.

If $V, W$ are vector subspaces of the matrix algebra $A$, we denote:

$$
\begin{gathered}
V+W=\{v+w: v \in V, w \in W\}, \\
V \cdot W=\operatorname{span}\{v w: v \in V, w \in W\}, \\
{[V, W]=\operatorname{span}\{v w-w v: v \in V, w \in W\} .}
\end{gathered}
$$

We recall the definition of a commuting square (see [Po1,, Po2]):

Definition 2.1. A commuting square of matrix algebras is a square of unital inclusions:

$$
\mathfrak{C}=\left(\begin{array}{ccc}
P_{-1} & \subset & P_{0} \\
\cup & & \cup \\
Q_{-1} & \subset & Q_{0}
\end{array}\right),
$$

where $P_{0}, P_{-1}, Q_{0}, Q_{-1}$ are matrix algebras and $\tau$ is a trace on $P_{0}, \tau(1)=1$, satisfying the condition:

$$
E_{P_{-1}} E_{Q_{0}}=E_{Q_{0}} E_{P_{-1}}=E_{Q_{-1}} .
$$

We say that the commuting square $\mathfrak{C}$ is non-degenerate if $P_{0}=P_{-1} Q_{0}$. We will assume all our commuting squares to be non-degenerate.

We now introduce the large second relative commutant (LRC) condition that we will consider.

Definition 2.2. Let $\mathfrak{C}$ be a commuting square with a $\lambda$ - Markov trace $\tau$. With the previous notation, let $\mathfrak{L}$ denote the lattice obtained by doing Jones' basic construction (see [JS]) from $\mathfrak{C}$ :

$$
\mathfrak{L}=\left(\begin{array}{ccccc}
P_{-1} & \subset & P_{0} & \stackrel{e}{\subset} & P_{1} \\
\cup & & \cup & & \cup \\
Q_{-1} & \subset & Q_{0} & \stackrel{e}{\subset} & Q_{1}
\end{array}\right),
$$


where the extension of the trace $\tau$ to $P_{1}$ is still denoted by $\tau$ and $e$ is the Jones projection of the basic construction. Let $R_{1}=P_{-1}^{\prime} \cap Q_{1}$ denote the second relative commutant associated to the commuting square $\mathfrak{C}$. We say that $\mathfrak{C}$ satisfies the LRC condition if the following two dual equalities hold:

$$
\begin{aligned}
& E_{Q_{1}}\left(P_{-1}^{\prime} \cap P_{1}\right)=R_{1}, \\
& E_{P_{0}}\left(R_{1}^{\prime} \cap P_{1}\right)=P_{-1} .
\end{aligned}
$$

Remark 2.3. For any $\mathfrak{C}$ we have: $R_{1}=E_{Q_{1}}\left(P_{-1}^{\prime} \cap Q_{1}\right) \subset E_{Q_{1}}\left(P_{-1}^{\prime} \cap P_{1}\right)$. Thus condition (11) can be interpreted as a largeness condition on $R_{1}$, hence the name LRC (large relative commutant).

Similarly, $P_{-1}=E_{P_{0}}\left(P_{-1}\right) \subset E_{P_{0}}\left(R_{1}^{\prime} \cap P_{1}\right)$, as $\left[P_{-1}, R_{1}\right]=0$. Thus, condition (2) also requires that $R_{1}$ be large, since it is a restriction on the size of its commutant.

Asking for a "largeness" condition on the relative commutant is inspired by the case of the standard invariant of a subfactor, as will be discussed in Section 5. We will see that in this context, (11) and (2) are equivalent and dual to each other.

Our main result, proved in Section 4, states:

Theorem 2.4. There exist only finitely many isomorphism classes of commuting squares $\mathfrak{C}$, with $\operatorname{dim}\left(P_{0}\right)$ fixed, satisfying the LRC condition.

We will prove the theorem by contradiction: assuming that there exist infinitely many such commuting squares, we find a convergent subsequence, to which we apply derivation techniques similar to those that we introduced in [Ni.

To make the notion of convergence of commuting squares precise, let us first recall the following definition and result from $[\mathrm{Chr}$ :

Definition 2.5. Let $A$ be a matrix algebra with normalized trace $\tau$. Denote $\mathcal{S}(A)=$ the set of all *-subalgebras of $A$ containing the identity. For $B_{1}, B_{2} \in \mathcal{S}(A)$ and $\delta>0$ we say that $B_{1}$ is $\delta$-contained in $B_{2}$ if for every element $x \in B_{1}$ of $\|x\|=1$ there exists $y \in B_{2}$ such that $\|x-y\|_{2}<\delta$. Here $\|\cdot\|_{2}$ denotes the norm given by the trace $\tau$ on $A$, i.e. $\|x\|_{2}=\tau\left(x^{*} x\right)^{1 / 2}$.

If $B_{1}$ is $\delta$-contained in $B_{2}$ and $B_{2}$ is $\delta$-contained in $B_{1}$ we write $\left\|B_{1}-B_{2}\right\|_{2, A}<\delta$.

Theorem 2.6. With the previous notation, there exists a continuous increasing function $f:[0, \infty) \rightarrow[0, \infty), f(0)=0$, such that if $\delta$ is small and $\left\|B_{1}-B_{2}\right\|_{2, A}<\delta$, then $B_{2}=\operatorname{Ad}(U)\left(B_{1}\right)$ for some unitary element $U \in A,\|U-I\|_{2}<f(\delta)$.

If Theorem 2.4 is false, then there exist infinitely many non-isomorphic LRC commuting squares

$$
\left.\mathfrak{C}_{n}=\left(\begin{array}{ccc}
P_{-1}^{n} & \subset & P_{0} \\
\cup & & \cup \\
Q_{-1}^{n} & \subset & Q_{0}^{n}
\end{array}\right), \tau\right) .
$$

By using Theorem 2.6 together with the compactness of the unit ball of $P_{1}$ it follows that the inclusions $Q_{-1}^{n} \subset P_{-1}^{n} \subset P_{0}$ are unitarily conjugate for infinitely many $n$. Thus, after conjugating each $\mathfrak{C}_{n}$ by a unitary and eventually passing to a subsequence, we may assume that

$$
\mathfrak{C}_{n}=\left(\begin{array}{ccc}
P_{-1} & \subset & P_{0} \\
\cup & & \cup \\
Q_{-1} & \subset & Q_{0}^{n}
\end{array}, \tau\right) .
$$


By a similar compactness argument, after passing to a subsequence we may assume that $Q_{0}^{n}=u_{n} Q_{1} u_{n}^{*}$, with $u_{n} \rightarrow I$ unitaries in $P_{0}$. We have $u_{n}^{*} Q_{-1} u_{n} \subset Q_{1}$ and Lemma 3.2 shows that $u_{n}=q_{n}^{\prime} q_{n}$ for $n$ large, with $q_{n} \rightarrow I$ unitaries in $Q_{0}$ and $q_{n}^{\prime} \rightarrow I$ unitaries in $Q_{-1}^{\prime} \cap P_{0}$. Since $u_{n} Q_{1} u_{n}^{*}=q_{n}^{\prime} q_{n} Q_{1} q_{n}^{*}\left(q_{n}^{\prime}\right)^{*}=q_{n}^{\prime} Q_{1}\left(q_{n}^{\prime}\right)^{*}$, by substituting $u_{n} \rightarrow q_{n}^{\prime}$ we may assume that $u_{n} \in Q_{-1}^{\prime} \cap P_{0}$.

In the following proposition we show that the notion of an LRC commuting square behaves well to limits. This is somewhat surprising, since it is not true in general that $P_{-1}^{\prime} \cap Q_{1}^{n}=R_{1}^{n}(n \geq 1)$ implies $P_{-1}^{\prime} \cap Q_{1}=R_{1}$. However, $P_{-1}^{\prime} \cap Q_{1}=R_{1}$ will follow from conditions (11), (2), which have nice continuity properties.

Proposition 2.7. With the previous notation, if $\mathfrak{C}_{n}$ are LRC commuting squares for all $n \geq 1$, then so is $\mathfrak{C}$.

Proof. If $B \subset A$ are matrix algebras and $u_{n} \rightarrow I$ are unitaries in $A$, then the conditional expectation satisfies the following continuity property:

$$
\lim _{n \rightarrow \infty} E_{u_{n} B u_{n}^{*}}(a)=E_{B}(a) \text {, for all } a \in A .
$$

This is easy to see since $E_{u_{n} B u_{n}^{*}}(a)=u_{n} E_{B}\left(u_{n}^{*} a u_{n}\right) u_{n}^{*}$.

We may assume the Jones projection $e$ to be the same for all the commuting squares $\mathfrak{C}_{n}$, i.e. the projection $e \in B\left(L^{2}\left(P_{0}, \tau\right)\right)$ implementing the conditional expectation $E_{P_{-1}}^{P_{0}}$. By the continuity of the conditional expectation, taking the limit of the relations $E_{P_{-1}} E_{Q_{0}^{n}}=E_{Q_{0}^{n}} E_{P_{-1}}=E_{Q_{-1}}$ implies that $\mathfrak{C}$ is a commuting square.

Let $P_{1}=\left\langle P_{0}, e\right\rangle, Q_{1}=\left\langle Q_{0}, e\right\rangle=\operatorname{span} Q_{0} e Q_{0}$ and $Q_{1}^{n}=\left\langle Q_{0}^{n}, e\right\rangle=\operatorname{span} Q_{0}^{n} e Q_{0}^{n} \subset$ $P_{1}$. Let $x_{1}, \ldots, x_{N}$ be a basis of $Q_{0}$. Then $u_{n} x_{k} u_{n}^{*} e u_{n} x_{l} u_{n}^{*}(1 \leq k, l \leq N) \operatorname{span} Q_{1}^{n}$, $x_{k} e x_{l}(1 \leq k, l \leq N)$ span $Q_{1}$ and $\left\|u_{n} x_{k} u_{n}^{*} e u_{n} x_{l} u_{n}^{*}-x_{k} e x_{l}\right\|_{2} \rightarrow 0$ as $n \rightarrow \infty$. The finiteness of the spanning set implies that $\left\|Q_{1}^{n}-Q_{1}\right\|_{2, P_{1}} \rightarrow 0$, so there exist unitaries $w_{n} \rightarrow I$ in $P_{1}$ such that $Q_{1}^{n}=w_{n} Q_{1} w_{n}^{*}$.

Let $R_{1}^{n}=P_{-1}^{\prime} \cap Q_{1}^{n}$. By eventually passing to a subsequence, we may assume that all $R_{1}^{n}$ are unitary conjugates inside $P_{1}$. By the compactness of the unit ball of $P_{1}$, after passing again to a subsequence, we may assume that these unitaries converge. It follows that $R_{1}^{n}=v_{n} R_{1} v_{n}^{*}$ with $v_{n} \rightarrow I$ unitaries in $P_{1}$, where $R_{1}$ is some subalgebra of $P_{1}$. Since $R_{1}^{n} \subset Q_{1}^{n}$ we have $R_{1} \subset Q_{1}$. However, it is not obvious that $P_{-1}^{\prime} \cap Q_{1}=R_{1}$ and in fact this will only follow because of the LRC conditions.

We know that $E_{Q_{1}^{n}}\left(P_{-1}^{\prime} \cap P_{1}\right)=R_{1}^{n}$ for all $n \geq 1$. Thus $v_{n}^{*} E_{w_{n} Q_{1} w_{n}^{*}}(x) v_{n} \in R_{1}$ for all $x \in P_{-1}^{\prime} \cap P_{1}$. From the continuity of the conditional expectation we obtain $E_{Q_{1}}\left(P_{-1}^{\prime} \cap P_{1}\right) \subset R_{1}$. On the other hand, $R_{1}^{n}=P_{-1}^{\prime} \cap Q_{1}^{n} \subset P_{-1}^{\prime} \cap P_{1}$ implies $\left[R_{1}, P_{-1}\right]=0$, so $R_{1} \subset P_{-1}^{\prime} \cap P_{1}$. Using this and the previous inclusion it follows that $E_{Q_{1}}\left(P_{-1}^{\prime} \cap P_{1}\right)=R_{1}$.

Notice that $R_{1} \subset P_{-1}^{\prime} \cap Q_{1}=E_{Q_{1}}\left(P_{-1}^{\prime} \cap Q_{1}\right) \subset E_{Q_{1}}\left(P_{-1}^{\prime} \cap P_{1}\right)=R_{1}$ also shows that $R_{1}=P_{-1}^{\prime} \cap Q_{1}$.

Since $E_{P_{0}}\left(\left(R_{1}^{n}\right)^{\prime} \cap P_{1}\right)=P_{-1}$ and $\left(R_{1}^{n}\right)^{\prime} \cap P_{1}=\left(v_{n} R_{1} v_{n}^{*}\right)^{\prime} \cap P_{1}=\left(v_{n} R_{1} v_{n}^{*}\right)^{\prime} \cap$ $\left(v_{n} P_{1} v_{n}^{*}\right)=v_{n}\left(R_{1}^{\prime} \cap P_{1}\right) v_{n}^{*}$, by taking the limit we obtain $E_{P_{0}}\left(R_{1}^{\prime} \cap P_{1}\right) \subset P_{-1}$. On the other hand, $\left[R_{1}, P_{-1}\right]=0$, so $P_{-1} \subset R_{1}^{\prime} \cap P_{1}$, which together with the previous inclusion yields $E_{P_{0}}\left(R_{1}^{\prime} \cap P_{1}\right)=P_{-1}$. This also implies that $P_{-1}=R_{1}^{\prime} \cap P_{0}$. 
Definition 2.8. We say that the sequence of unitaries $u_{n} \rightarrow I, u_{n} \neq I$, has direction of convergence $h$ if

$$
h=\lim _{n \rightarrow \infty} \frac{u_{n}-I}{i\left\|u_{n}-I\right\|} .
$$

Let $v_{n} \rightarrow I, v_{n} \neq I(n \geq 1)$ be a sequence of unitaries in $P_{0}$. Write $v_{n}=\exp \left(i k_{n}\right)$, with $k_{n} \in P_{0}$ hermitian non-zero elements, $k_{n} \rightarrow 0$. Consider any subsequence $\left(h_{n}\right)_{n \geq 1}$ of $\left(k_{n}\right)_{n \geq 1}$ such that $\frac{h_{n}}{\left\|h_{n}\right\|}$ converges to some norm one hermitian element $h$ of $P_{0}$. Such sequences $\left(h_{n}\right)_{n \geq 1}$ exist by a standard compactness argument and any such $h$ will be referred to as a direction of convergence for $\left(v_{n}\right)_{n \geq 1}$. This terminology is compatible with the previous definition, as $h$ is the direction of convergence of the subsequence $u_{n}=\exp \left(i h_{n}\right)$ of $\left(v_{n}\right)_{n \geq 1}$. Indeed:

$$
h=\lim _{n \rightarrow \infty} \frac{u_{n}-I}{i\left\|h_{n}\right\|}=\lim _{n \rightarrow \infty} \frac{u_{n}-I}{i\left\|u_{n}-I\right\|} .
$$

We end this section by recalling a result from [Ni], which gives normalizations on the direction of convergence of a sequence of commuting squares. These will be essential for the proof of Theorem 2.4.

Proposition 2.9. Let $P_{0}$ be a matrix algebra with trace $\tau$ and let

$$
\mathfrak{C}_{n}^{*}=\left(\begin{array}{ccc}
P_{-1} & \subset & P_{0} \\
\cup & & \cup \\
Q_{-1} & \subset & u_{n} Q_{0} u_{n}^{*}
\end{array}\right)
$$

be a sequence of non-isomorphic commuting squares, where $u_{n} \rightarrow I$ are unitaries in $Q_{-1}^{\prime} \cap P_{0}$. After eventually replacing $u_{n}$ by one of its subsequences, we have:

There exist unitaries $q_{n} \in Q_{-1}^{\prime} \cap Q_{0}, q_{n}^{\prime} \in Q_{0}^{\prime} \cap P_{-1}, p_{n} \in Q_{-1}^{\prime} \cap P_{-1}, p_{n}^{\prime} \in P_{-1}^{\prime} \cap P_{0}$ such that:

$$
\begin{gathered}
\tilde{u_{n}}=p_{n} p_{n}{ }^{\prime} u_{n} q_{n}^{\prime} q_{n} \rightarrow I, \\
\lim _{n \rightarrow \infty} \frac{\tilde{u_{n}}-I}{i\left\|\tilde{u_{n}}-I\right\|}=\tilde{h} \in Q_{-1}^{\prime} \cap P_{0}, \\
E_{P_{-1}^{\prime} \cap P_{0}}(\tilde{h})=E_{Q_{0}^{\prime} \cap P_{0}}(\tilde{h})=E_{Q_{-1}^{\prime} \cap P_{-1}}(\tilde{h})=E_{Q_{-1}^{\prime} \cap Q_{0}}(\tilde{h})=0 .
\end{gathered}
$$

Remark 2.10. Note that the change $u_{n} \rightarrow \tilde{u_{n}}=p_{n} p_{n}{ }^{\prime} u_{n} q_{n}^{\prime} q_{n}$ preserves the isomorphism class of the commuting square $\mathfrak{C}_{n}$.

\section{LOCAL MINIMA OF MATRIX ALGEBRA INTERSECTIONS}

In this section we deal with the main technical ingredient of the paper. Let $C$ be a matrix algebra and $A, B$ two *-closed subalgebras of $C$. Consider the algebras $A \cap w_{n} B w_{n}^{*}$, where $w_{n}$ are unitaries in $C$ approaching the identity. We find restrictions on the directions of convergence of $w_{n}$ such that $A \cap B$ can be unitarily embedded in $A \cap w_{n} B w_{n}^{*}$ for all $n$ large. In other words, we study when $A \cap B$ is what one might call a local minimum along the curve $A \cap w B w^{*}$, with $w \rightarrow I$ unitaries in the direction $h$.

In the subsequent results we will often use the following relation that holds true for every $a, b, c$ in a matrix algebra $(C, \tau)$ :

$$
\tau([a, b] c)=\tau(a[b, c])=\tau([c, a] b),
$$


as it can easily be checked that $\tau([a, b] c)=\tau(a b c-b a c)=\tau(a b c)-\tau(b a c)=$ $\tau(a b c)-\tau(a c b)=\tau(a[b, c])=\tau(c a b)-\tau(a c b)$.

Since a lot of the derivatives of the relations we will consider are commutator relations, the following lemma will be very useful.

Lemma 3.1. Let $(C, \tau)$ be a matrix algebra with a trace $\tau$. Let $A \subset B$ be $*$-closed subalgebras of $C$. For $c \in C$ we have:

$$
[c, A] \subset B \text { if and only if } c \in B+A^{\prime} \cap C .
$$

Proof. The right to left implication is clearly true, as

$$
\left[B+A^{\prime} \cap C, A\right] \subset[B, A]+\left[A^{\prime} \cap C, A\right]=[B, A] \subset[B, B] \subset B .
$$

Assume that $c$ is such that $[c, A] \subset B$. Let $c_{0}=E_{B}(c) \in B$. Since $\left[c_{0}, A\right] \subset$ $[B, B] \subset B$, we have $\left[c-c_{0}, A\right] \subset B$. On the other hand, $\left[c-c_{0}, A\right]$ is perpendicular to $B$. Indeed, for $a \in A$ and $b \in B$, equation (3) implies:

$$
\tau\left(\left[c-c_{0}, a\right] b^{*}\right)=\tau\left(\left(c-c_{0}\right)\left[a, b^{*}\right]\right)=0
$$

as $\left[a, b^{*}\right] \in B$ and $c-c_{0}$ is orthogonal to $B$.

It follows that $\left[c-c_{0}, A\right]=0$, so $c-c_{0} \in A^{\prime} \cap C$. Thus $c \in B+A^{\prime} \cap C$, which ends the proof.

A consequence of Lemma 3.1 is the following:

Lemma 3.2. Let $(C, \tau)$ be a matrix algebra with a trace $\tau$. Let $A \subset B$ be $*$-closed subalgebras of $C$. Let $c_{n} \rightarrow I$ be unitaries in $C$ such that $c_{n} A c_{n}^{*} \subset B$ for all $n$. Then there exist unitaries $b_{n} \rightarrow I$ in $B$ and $a_{n}^{\prime} \rightarrow I$ in $A^{\prime} \cap C$ such that $c_{n}=b_{n} a_{n}$ for all $n$ large.

Proof. Let $\mathfrak{X}=\mathcal{U}(B) \times \mathcal{U}\left(A^{\prime} \cap C\right)$. Since $\mathfrak{X}$ is compact in \|\|$_{2}$, for every $n$ there exist elements $b_{n} \in B, a_{n}^{\prime} \in A^{\prime} \cap C$ that realize the minimum:

$$
\left\|b_{n}^{*} c_{n} a_{n}^{* *}-I\right\|_{2}=\inf _{\left(b, a^{\prime}\right) \in \mathfrak{X}}\left\|b^{*} c_{n} a^{\prime *}-I\right\|_{2} .
$$

Let $u_{n}=b_{n}^{*} c_{n}\left(a_{n}^{\prime}\right)^{*}$. Clearly $u_{n} \rightarrow I$, since for $b=a^{\prime}=I$ we have $\left\|u_{n}-I\right\|_{2} \leq$ $\left\|c_{n}-I\right\|_{2}$. If $u_{n}=I$ for all $n$ large, we are done. Assume, by eventually passing to a subsequence, that $u_{n} \neq I$ for all $n$. By passing again to a subsequence, we may assume that $h=\lim _{n \rightarrow \infty} \frac{u_{n}-I}{i\left\|u_{n}-I\right\|}$ exists. Clearly $\|h\|=1$.

Let $\Re \tau$ denote the real part of the trace $\tau$. For every $u$ unitary we have $\|u-I\|_{2}^{2}=$ $2-2 \Re \tau(u)$. It follows that

$$
\Re \tau\left(u_{n}\right) \geq \Re \tau\left(b^{*} c_{n} a^{\prime *}\right), \text { for all }\left(b, a^{\prime}\right) \in \mathfrak{X} .
$$

Let $\lambda$ be a real number, let $b_{0} \in B$ be a hermitian element, and let $b=$ $\left(\exp \left(i \lambda b_{0}\right) b_{n}^{*}\right)^{*}, a^{\prime}=a_{n}^{\prime}$. The previous inequality implies:

$$
\Re \tau\left(u_{n}\right) \geq \Re \tau\left(\exp \left(i \lambda b_{0}\right) u_{n}\right) \Longrightarrow \Re \tau\left(\left(\exp \left(i \lambda b_{0}\right)-I\right) u_{n}\right) \leq 0 .
$$

After dividing by $\lambda>0$ and taking the limit as $\lambda$ approaches 0 , we obtain $\Re \tau\left(i b_{0} u_{n}\right) \leq 0$. Similarly, after dividing by $\lambda<0$ we have $\Re \tau\left(i b_{0} u_{n}\right) \geq 0$. It follows that

$$
\Re \tau\left(i b_{0} u_{n}\right)=0 .
$$

Since for hermitians $b_{0}$ we have $\Re \tau\left(i b_{0}\right)=0$, we can rewrite the previous equality as $\Re \tau\left(i b_{0}\left(u_{n}-I\right)\right)=0$ and after dividing by $\left\|u_{n}-I\right\|$ and taking the limit we obtain 
$\Re \tau\left(b_{0} h\right)=0$. Since $\tau\left(b_{0} h\right)=\tau\left(h b_{0}\right)=\tau\left(\left(b_{0} h\right)^{*}\right)$, it follows that $\tau\left(b_{0} h\right)$ is a real number and thus $\tau\left(b_{0} h\right)=0$. Consequently:

$$
E_{B}(h)=0 .
$$

Similar arguments show that $E_{A^{\prime} \cap C}(h)=0$. Also, note that $u_{n} A u_{n}^{*} \subset B$ for all $n \geq 1$.

For every $a \in A$ we have $\left(u_{n}-I\right) a u_{n}^{*}+a\left(u_{n}-I\right)=u_{n} a u_{n}^{*}-a \in B$ for all $n \geq 1$. After dividing by $i\left\|u_{n}-I\right\|$ and taking the limit, we obtain

$$
[h, a] \in B \text { for all } a \in A \text {, }
$$

and Lemma 3.1 implies

$$
h \in B+\left(A^{\prime} \cap C\right) .
$$

Since the vector space $B+\left(A^{\prime} \cap C\right)$ can be written as the sum of two orthogonal subspaces $\left(B \ominus\left(A^{\prime} \cap B\right)\right) \oplus\left(A^{\prime} \cap C\right)$ and $h$ is orthogonal on both of these subspaces, we obtain $h=0$, which is a contradiction. Thus, $u_{n}=I$ for all $n$ large, or equivalently $c_{n}=b_{n} a_{n}$.

We now present the main result of this section, dealing with local minima of intersections of algebras.

Proposition 3.3. Let $(C, \tau)$ be a matrix algebra with a trace $\tau$. Let $A, B$ be *closed subalgebras of $C$. Let $w_{n} \rightarrow I$ be a sequence of unitaries in $C$ such that $w_{n} \neq I$ and $h=\lim _{n \rightarrow \infty} \frac{w_{n}-I}{i\left\|w_{n}-I\right\|}$ exists. Assume that $A \cap B$ unitarily embeds into $A \cap w_{n} B w_{n}^{*}$ for all $n \geq 1$. Then

$$
h \in A+B+(A \cap B)^{\prime} \cap C .
$$

Proof. Let $v_{n} \rightarrow I$ be unitaries in $C$ such that

$$
v_{n}(A \cap B) v_{n}^{*} \subset A \cap w_{n} B w_{n}^{*}
$$

for all $n \geq 1$. After eventually passing to a subsequence we may assume that $v_{n} \rightarrow v \in C$. By taking the limit it follows that $v(A \cap B) v^{*} \subset A \cap B$ and because both sides have the same dimension we must have equality. Then $\tilde{v}_{n}=v_{n} v^{*} \rightarrow I$ and $\tilde{v}_{n}(A \cap B) \tilde{v}_{n}^{*}=v_{n}(A \cap B) v_{n}^{*} \subset A \cap w_{n} B w_{n}^{*}$. This shows that we can assume, by substituting $v_{n} \rightarrow \tilde{v}_{n}$, that $v_{n} \rightarrow I$.

Assume that infinitely many of the $v_{n}$ are different from $I$. By eventually passing to a subsequence, we may assume that the $\operatorname{limit} \tilde{h}=\lim _{n \rightarrow \infty} \frac{v_{n}-I}{i\left\|v_{n}-I\right\|}$ exists. Notice that by modifying $v_{n} \rightarrow v_{n} s_{n} s_{n}^{\prime}$ with unitaries $s_{n} \in A \cap B, s_{n}^{\prime} \in(A \cap B)^{\prime} \cap C$, $s_{n}, s_{n}^{\prime} \rightarrow I$, we do not change the algebra $v_{n}(A \cap B) v_{n}^{*}$. Thus, an argument similar to Proposition 2.9 shows that we may assume $\tilde{h}$ orthogonal to $A \cap B$ and $(A \cap B)^{\prime} \cap C$.

For $n \geq 1$ let $r_{n}=\sup \left(\left\|w_{n}-I\right\|,\left\|v_{n}-I\right\|\right)$. Clearly $r_{n} \neq 0$. By eventually passing to a subsequence, we may assume that the following limits exist:

$$
h_{w}=\lim _{n \rightarrow \infty} \frac{w_{n}-I}{i r_{n}}, \quad h_{v}=\lim _{n \rightarrow \infty} \frac{v_{n}-I}{i r_{n}} .
$$

Notice that from the definition of $r_{n}$ it follows that at least one of $h_{w}, h_{v}$ must be non-zero.

Since $\frac{\left\|w_{n}-I\right\|}{r_{n}} \cdot \frac{w_{n}-I}{i\left\|w_{n}-I\right\|}=\frac{w_{n}-I}{i r_{n}}$, we have $h_{w}=c h$ for some positive scalar $c$ (which may be 0 ). In particular, $h_{w}$ is hermitian. A similar argument shows that $h_{v}=d \tilde{h}$ for some $d \geq 0$; thus $h_{v}$ is hermitian and orthogonal to $A \cap B,(A \cap B)^{\prime} \cap C$. 
Let $s \in A \cap B$. We have $\left(v_{n}-I\right) s v_{n}^{*}+s\left(v_{n}-I\right)^{*}=v_{n} s v_{n}^{*}-s \in A$. Consequently:

$$
\frac{v_{n}-I}{i r_{n}} s v_{n}^{*}-s\left(\frac{v_{n}-I}{i r_{n}}\right)^{*} \in A .
$$

After taking the limit of this relation as $n \rightarrow \infty$ and using $h_{v}=h_{v}^{*}$, we obtain

$$
\left[h_{v}, s\right] \in A \text { for all } s \in A \cap B,
$$

and Lemma 3.1 implies

$$
h_{v} \in A+(A \cap B)^{\prime} \cap C .
$$

For $s \in A \cap B$ we also have $v_{n} s v_{n}^{*} \in w_{n} B w_{n}^{*}$. Equivalently:

$$
w_{n}^{*} v_{n} s\left(w_{n}^{*} v_{n}\right)^{*} \in B \text { for all } n \geq 1 \text {. }
$$

Observe that

$$
\frac{w_{n}^{*} v_{n}-I}{i r_{n}}=-\left(\frac{w_{n}-I}{i r_{n}}\right)^{*} v_{n}+\frac{v_{n}-I}{i r_{n}} \rightarrow h_{v}-h_{w} .
$$

Thus, after dividing by $i r_{n}$ and taking the limit, equation (5) yields

$$
\left[h_{v}-h_{w}, s\right] \in B \text { for all } s \in A \cap B,
$$

and after applying Lemma 3.1 again we obtain

$$
h_{v}-h_{w} \in B+(A \cap B)^{\prime} \cap C .
$$

Combining (4) and (6) yields

$$
h_{w} \in A+B+(A \cap B)^{\prime} \cap C .
$$

Since $h_{w}=c h$, we only need to argue that $c \neq 0$ to finish the proof.

If $c=0$, then $h_{w}=0$ and equation (6) becomes

$$
h_{v} \in B+(A \cap B)^{\prime} \cap C .
$$

Thus $h_{v}=b+s^{\prime}$ with $b \in B$ and $s^{\prime} \in(A \cap B)^{\prime} \cap C$. We have

$$
0=E_{(A \cap B)^{\prime} \cap C}\left(h_{v}\right)=E_{(A \cap B)^{\prime} \cap C}(b)+s^{\prime},
$$

which implies $s^{\prime}=-E_{(A \cap B)^{\prime} \cap C}(b) \in(A \cap B)^{\prime} \cap B$, so $h_{v}=b+s^{\prime} \in B$.

Similarly, equation (4) implies $h_{v} \in A$. Thus $h_{v} \in A \cap B$, which implies $h_{v}=0$ since $h_{v}$ is orthogonal to $A \cap B$. We have thus obtained that both $h_{v}, h_{w}$ are 0 , which is impossible.

We still have to deal with the case when infinitely many $v_{n}$ are equal to $I$. This yields

$$
A \cap B \subset A \cap w_{n} B w_{n}^{*} .
$$

Thus for every $s \in A \cap B$ we have

$$
\left(w_{n}-I\right) s w_{n}^{*}+s\left(w_{n}-I\right)^{*}=w_{n}^{*} s w_{n}-s \in B .
$$

Dividing by $i\left\|w_{n}-I\right\|$ and taking the limit yields $[h, s] \in B$ for all $s \in A \cap B$. Thus in this case we easily obtain $h \in B+(A \cap B)^{\prime} \cap C$.

Remark 3.4. Similar arguments as in Lemma 3.2 can be used to show that $w_{n}=$ $a_{n} s_{n} b_{n}$, with $a_{n} \in A, b_{n} \in B, s_{n} \in(A \cap B)^{\prime} \cap C$. However, to deduce the conclusion of the proposition, one still needs a proof along the same lines to control the speed of convergence of $a_{n}, b_{n}, s_{n}$.

We end this section with a lemma that will be useful towards proving the main result. 
Lemma 3.5. Let $(C, \tau)$ be a matrix algebra with a trace $\tau$. Let $A, B$ be $*$-closed subalgebras of $C$ and $D=A \cap B$. Assume that the commuting square condition holds: $E_{A} E_{B}=E_{B} E_{A}=E_{D}$. Then we have the following equality of vector spaces:

$$
D^{\prime} \cap(A+B)=D^{\prime} \cap A+D^{\prime} \cap B .
$$

Proof. We just have to show that " $\subset$ " holds. Let $a \in A, b \in B$ be such that $a+b \in D^{\prime} \cap C$. Since $D \subset A \subset C$, we have $E_{A}\left(D^{\prime} \cap C\right)=D^{\prime} \cap A$. On the other hand, $E_{A}(a+b)=a+E_{A}(b)=a+E_{D}(b)$. It follows that $a+E_{D}(b) \in D^{\prime} \cap A$. If we rewrite $a+b=a_{1}+b_{1}$, with $a_{1}=a+E_{D}(b) \in A$ and $b_{1}=b-E_{D}(b) \in B$, we have $a_{1} \in D^{\prime} \cap C$, which also implies $b_{1} \in D^{\prime} \cap C$. This ends the proof.

\section{THE MAIN RESUlT}

We are now ready to prove Theorem 2.4, stating that there exist only finitely many isomorphism classes of commuting squares $\mathfrak{C}$, with $\operatorname{dim}\left(P_{0}\right)$ fixed, satisfying the $L R C$ condition. Since we fix $\operatorname{dim}\left(P_{0}\right)$, it is clear that we may in fact assume that both the algebra $P_{0}$ and the $\lambda$-Markov trace $\tau$ are fixed, without changing the finiteness result.

We assume, by contradiction, that the theorem is false. The discussion from Section 2 then shows that there exist non-isomorphic commuting squares

$$
\mathfrak{C}_{n}=\left(\begin{array}{ccc}
P_{-1} & \subset & P_{0} \\
\cup & & \cup \\
Q_{-1} & \subset & u_{n} Q_{0} u_{n}^{*}
\end{array}, \tau\right), \mathfrak{C}=\left(\begin{array}{ccc}
P_{-1} & \subset & P_{0} \\
\cup & & \cup \\
Q_{-1} & \subset & Q_{0}
\end{array}\right)
$$

all satisfying the $L R C$ condition, where $u_{n} \rightarrow I$ are unitaries in $Q_{-1}^{\prime} \cap P_{0}, u_{n} \neq I$. We may also assume, by eventually passing to a subsequence, that the unitaries $u_{n}$ converge in the direction $h_{0}$, i.e.

$$
h_{0}=\lim _{n \rightarrow \infty} \frac{u_{n}-I}{i\left\|u_{n}-I\right\|} .
$$

Proposition 2.9 shows that we may take $h_{0}$ orthogonal to $Q_{-1}^{\prime} \cap Q_{0}, Q_{-1}^{\prime} \cap P_{-1}, P_{-1}^{\prime} \cap$ $P_{0}, Q_{0}^{\prime} \cap P_{0}$.

Moreover, we may assume that the lattices $\mathfrak{L}_{n}, \mathfrak{L}$ obtained by doing the basic construction from $\mathfrak{C}_{n}, \mathfrak{C}$ are of the form:

$$
\begin{gathered}
\mathfrak{L}_{n}=\left(\begin{array}{ccccc}
P_{-1} & \subset & P_{0} & \stackrel{e}{\subset} & P_{1} \\
\cup & & \cup & & \cup \\
Q_{-1} & \subset & u_{n} Q_{0} u_{n}^{*} & \stackrel{e}{\subset} & w_{n} Q_{1} w_{n}^{*}
\end{array}, \tau\right), \\
\mathfrak{L}=\left(\begin{array}{ccccc}
P_{-1} & \subset & P_{0} & \stackrel{e}{\subset} & P_{1} \\
\cup & & \cup & & \cup \\
Q_{-1} & \subset & Q_{0} & \stackrel{e}{\subset} & Q_{1}
\end{array}\right),
\end{gathered}
$$

where $e$ is the Jones projection of the basic construction $P_{-1} \subset P_{0} \subset P_{1}, u_{n} \rightarrow I$ are unitaries in $P_{0}$ and $w_{n} \rightarrow I$ are unitaries in $P_{1}$. Also, $R_{1}^{n}=P_{-1}^{\prime} \cap w_{n} Q_{1} w_{n}^{*}$ is unitarily conjugate to $R_{1}=P_{-1} \cap Q_{1}$ for all $n$.

Since $P_{0} \cap w_{n} Q_{1} w_{n}^{*}=u_{n} Q_{0} u_{n}^{*}$, it is clear that $w_{n} \neq I$ for $n$ large. By eventually passing to a subsequence, we may assume the existence of the limit

$$
h_{1}=\lim _{n \rightarrow \infty} \frac{w_{n}-I}{i\left\|w_{n}-I\right\|} .
$$


Notice that the algebra $w_{n} Q_{1} w_{n}^{*}$ does not change if we modify $w_{n}$ by multiplying it on the right with unitaries of $Q_{1}$ or $Q_{1}^{\prime} \cap P_{0}$. Thus, similar arguments to Proposition 2.9 and Lemma 3.2 show that we may assume $h_{1}$ is orthogonal to $Q_{1}, Q_{1}^{\prime} \cap P_{1}$.

Applying Proposition 3.3 for $A=P_{-1}^{\prime} \cap P_{1}, B=Q_{1}, R_{1}=A \cap B$ yields

$$
h_{1} \in P_{-1}^{\prime} \cap P_{1}+Q_{1}+R_{1}^{\prime} \cap P_{1} .
$$

For $n \geq 1$ let $r_{n}=\sup \left(\left\|w_{n}-I\right\|,\left\|u_{n}-I\right\|\right)$. Clearly $r_{n} \neq 0$. By eventually passing to a subsequence, we may assume that the following limits exist:

$$
h_{w}=\lim _{n \rightarrow \infty} \frac{w_{n}-I}{i r_{n}}, \quad h_{u}=\lim _{n \rightarrow \infty} \frac{u_{n}-I}{i r_{n}} .
$$

Notice that from the definition of $r_{n}$ it follows that at least one of $h_{w}, h_{u}$ must be non-zero. Also, arguments similar to those from Proposition 3.3 show that $h_{u}=c h_{0}, h_{w}=d h_{1}$ for some positive (but possibly equal to zero!) scalars $c, d$. It follows that $h_{u}, h_{v}$ also satisfy

$$
\begin{gathered}
h_{u} \in Q_{-1}^{\prime} \cap P_{0}, \\
h_{w} \in P_{-1}^{\prime} \cap P_{1}+Q_{1}+R_{1}^{\prime} \cap P_{1}, \\
h_{u} \perp Q_{-1}^{\prime} \cap Q_{0}, Q_{-1}^{\prime} \cap P_{-1}, P_{-1}^{\prime} \cap P_{0}, Q_{0}^{\prime} \cap P_{0}, \\
h_{w} \perp Q_{1}, Q_{1}^{\prime} \cap P_{1} .
\end{gathered}
$$

We have

$$
\lim _{n \rightarrow \infty} \frac{w_{n}^{*} u_{n}-I}{i r_{n}}=h_{u}-h_{w} .
$$

Since $u_{n} Q_{0} u_{n}^{*} \subset w_{n} Q_{1} w_{n}^{*}$, it follows that $\left(w_{n}^{*} u_{n}\right) Q_{0}\left(w_{n}^{*} u_{n}\right)^{*} \subset Q_{1}$ for all $n$. Thus

$$
\left(w_{n}^{*} u_{n}-I\right) q_{0}\left(w_{n}^{*} u_{n}\right)^{*}+q_{0}\left(w_{n}^{*} u_{n}-I\right)^{*} \in Q_{1} \text { for all } q_{0} \in Q_{0} .
$$

After dividing by $i r_{n}$, using (11) and taking the limit we obtain

$$
\left[h_{u}-h_{w}, q_{0}\right] \in Q_{1} \text { for all } q_{0} \in Q_{0} .
$$

Using Lemma 3.1 yields

$$
h_{u}-h_{w} \in Q_{1}+Q_{0}^{\prime} \cap P_{1} .
$$

Thus, we may write $h_{w}=h_{u}+h$, where $h$ is a hermitian in $Q_{1}+Q_{0}^{\prime} \cap P_{1}$.

After projecting on $P_{0}$ and using the LRC condition $E_{P_{0}}\left(R_{1}^{\prime} \cap P_{1}\right)=P_{-1}$, equation (8) yields

$$
E_{P_{0}}\left(h_{w}\right) \in P_{-1}^{\prime} \cap P_{0}+Q_{0}+P_{-1} .
$$

On the other hand, $E_{P_{0}}\left(h_{w}\right)=h_{u}+E_{P_{0}}(h)$ and $E_{P_{0}}(h) \in E_{P_{0}}\left(Q_{1}\right)+E_{P_{0}}\left(Q_{0}^{\prime} \cap P_{1}\right) \subset$ $Q_{0}+Q_{0}^{\prime} \cap P_{0}$, which implies

$$
h_{u} \in P_{-1}^{\prime} \cap P_{0}+Q_{0}+P_{-1}+Q_{0}^{\prime} \cap P_{0} .
$$

We also know that $h_{u} \in Q_{-1}^{\prime} \cap P_{0}$. Using the previous relation and Lemma 3.5 for $A=Q_{0}, B=P_{-1}, D=Q_{-1}$, we obtain

$$
h_{u} \in P_{-1}^{\prime} \cap P_{0}+Q_{-1}^{\prime} \cap Q_{0}+Q_{-1}^{\prime} \cap P_{-1}+Q_{0}^{\prime} \cap P_{0} .
$$

This together with equation (9) implies $h_{u}=0$. Thus

$$
h_{w}=h \in Q_{1}+Q_{0}^{\prime} \cap P_{1} .
$$


We can write

$$
h_{w} \in Q_{1}+Q_{0}^{\prime} \cap P_{1}=Q_{1} \ominus\left(Q_{0}^{\prime} \cap Q_{1}\right) \oplus Q_{0}^{\prime} \cap P_{1},
$$

and the two vector spaces are orthogonal: $Q_{1} \ominus\left(Q_{0}^{\prime} \cap Q_{1}\right) \perp Q_{0}^{\prime} \cap P_{1}$. On the other hand, we know that $h_{w} \perp Q_{1}$. We obtain

$$
h_{w} \in Q_{0}^{\prime} \cap P_{1} .
$$

We now use the existence of the Jones projection $e \in w_{n} Q_{1} w_{n}^{*}$. Since $\left(w_{n}-\right.$ $I)^{*} e w_{n}+e\left(w_{n}-I\right)=w_{n}^{*} e w_{n}-e \in Q_{1}$ for all $n$, after dividing by $i r_{n}$ and taking the limit as $n \rightarrow \infty$ we obtain

$$
\left[h_{w}, e\right] \in Q_{1}
$$

However, $E_{Q_{1}}\left(\left[h_{w}, e\right]\right)=\left[E_{Q_{1}}\left(h_{w}\right), e\right]=0$, which shows that $\left[h_{w}, e\right]=0$. Thus $h_{w} \in e^{\prime} \cap P_{1}$. Together with equation (13) this yields

$$
h_{w} \in Q_{0}^{\prime} \cap e^{\prime} \cap P_{1}=\left\langle Q_{0}, e\right\rangle^{\prime} \cap P_{1}=Q_{1}^{\prime} \cap P_{1},
$$

which together with (10) implies $h_{w}=0$.

We have thus obtained that both $h_{v}, h_{w}$ are 0 , which is a contradiction.

\section{EXAMPLES}

Let $N \subset M$ be a subfactor of depth 2, i.e. $N^{\prime} \cap M \subset N^{\prime} \cap M_{1} \subset N^{\prime} \cap M_{2}$ is a basic construction. By a result of [Po1, $[\mathrm{Oc}$, the commuting square

$$
\mathfrak{C}=\left(\begin{array}{ccc}
N^{\prime} \cap M_{1} & \subset & N^{\prime} \cap M_{2} \\
\cup & & \cup \\
M^{\prime} \cap M_{1} & \subset & M^{\prime} \cap M_{2}
\end{array}\right)
$$

uniquely determines the isomorphism class of $N \subset M$. $\mathfrak{C}$ is called a standard commuting square. If we let $P_{0}=N^{\prime} \cap M_{2}, P_{-1}=N^{\prime} \cap M_{1}, Q_{0}=M^{\prime} \cap M_{2}, Q_{-1}=$ $M^{\prime} \cap M_{1}$ we have $P_{1}=\left\langle P_{0}, e_{3}\right\rangle=N^{\prime} \cap M_{3}$ and $Q_{1}=\left\langle Q_{0}, e_{3}\right\rangle=M^{\prime} \cap M_{3}$.

We have $P_{-1}^{\prime} \cap Q_{1}=\left(N^{\prime} \cap M_{1}\right)^{\prime} \cap\left(N^{\prime} \cap M_{3}\right)=M_{1}^{\prime} \cap M_{3}$. The last equality is easy to check, since $\left(N^{\prime} \cap M_{1}\right)^{\prime} \cap\left(N^{\prime} \cap M_{3}\right) \subset e_{1}^{\prime} \cap\left(M^{\prime} \cap M_{3}\right)=\left\langle M, e_{1}\right\rangle^{\prime} \cap M_{3}=M_{1} \cap M_{3}$ and clearly $M_{1}^{\prime} \cap M_{3} \subset\left(N^{\prime} \cap M_{1}\right)^{\prime} \cap\left(N^{\prime} \cap M_{3}\right)$. Thus:

$$
R_{1}=P_{-1}^{\prime} \cap Q_{1}=M_{1}^{\prime} \cap M_{3},
$$

which is anti-isomorphic (and therefore isomorphic) to $N^{\prime} \cap M_{1}$. This justifies asking for a largeness condition on $R_{1}$.

We now investigate when does $\mathfrak{C}$ satisfy the $L R C$ condition.

Proposition 5.1. Let $\mathfrak{C}$ be the standard commuting square associated to the depth 2 subfactor $N \subset M$. Then $\mathfrak{C}$ is an LRC commuting square if and only if

$$
E_{\left(N^{\prime} \cap M_{1}\right)^{\prime} \cap\left(N^{\prime} \cap M_{2}\right)}\left(e_{2}\right) \in \mathbb{C} I .
$$

Proof. Because of the duality in the lattice of relative commutants of a subfactor, conditions (11) and (2) from the definition of $L R C$ commuting squares are equivalent for $\mathfrak{C}$. Indeed, if $J$ is the conjugation map on $L^{2}\left(M_{1}, \tau\right)$ and we embed $N, M, M_{1}, M_{2}, M_{3}$ in $B\left(L^{2}\left(M_{1}, \tau\right)\right.$, we have $J P_{-1} J=R_{1}, J P_{0} J=Q_{1}, J P_{1} J=P_{1}$. This shows that

$$
E_{P_{0}}\left(R_{1}^{\prime} \cap P_{1}\right)=P_{-1} \text { if and only if } E_{Q_{1}}\left(P_{-1}^{\prime} \cap P_{0}\right)=R_{1} .
$$


Is is thus sufficient to work with $E_{Q_{1}}\left(P_{-1}^{\prime} \cap P_{0}\right)=R_{1}$. Equivalently, this can be written as

$$
Q_{1} \ominus R_{1} \perp P_{-1}^{\prime} \cap P_{1} .
$$

Assume (15) holds. Let $\lambda^{-1}=[M: N]$. Since $e_{2} \in Q_{1}$ and $E_{R_{1}}\left(e_{2}\right)=\lambda \cdot I$, we have $e_{2}-\lambda I \in Q_{1} \ominus R_{1}$ and thus we must have $e_{2}-\lambda I \perp P_{-1}^{\prime} \cap P_{1}$. Equivalently, $E_{P_{-1}^{\prime} \cap P_{1}}\left(e_{2}\right)=\lambda I$. Since $e_{2} \in P_{0}$, we have

$$
E_{P_{-1}^{\prime} \cap P_{0}}\left(e_{2}\right)=E_{P_{-1}^{\prime} \cap P_{1}}\left(e_{2}\right)=\lambda I \in \mathbb{C} I,
$$

which shows that the left to right implication holds true.

We now prove that $E_{P_{-1}^{\prime} \cap P_{0}}\left(e_{2}\right)=\lambda I$ implies (15)). Let $x \in P_{-1}^{\prime} \cap P_{1}$. It is sufficient to show that $y=x-E_{R_{1}}(x) \in P_{-1}^{\prime} \cap P_{1}$ is orthogonal to $Q_{1}$. Since $Q_{1}=\left\langle R_{1}, e_{2}\right\rangle$, it is enough to show that $y$ is orthogonal on elements of the form $r e_{2} r^{\prime}$, with $r, r^{\prime} \in R_{1}$. We have $\tau\left(\right.$ yre $\left._{2} r^{\prime}\right)=\tau\left(r^{\prime} y r e_{2}\right)$. Since $r^{\prime} y r \in P_{-1}^{\prime} \cap P_{1}$ and $E_{P_{-1}^{\prime} \cap P_{1}}\left(e_{2}\right)=E_{P_{-1}^{\prime} \cap P_{0}}\left(e_{2}\right)=\lambda I$, we obtain

$$
\tau\left(\text { yre }_{2} r^{\prime}\right)=\tau\left(r^{\prime} y r e_{2}\right)=\lambda \tau\left(r^{\prime} y r\right)=\lambda \tau\left(y r r^{\prime}\right)=0
$$

as $y$ is orthogonal to $R_{1}$. This ends the proof.

By combining the previous result with Theorem 2.4. we obtain the following finiteness result for the standard invariants of finite depth subfactors. We mention that this result is well known to specialists as a theorem of A. Ocneanu, even without the extra assumption $E_{\left(N^{\prime} \cap M_{1}\right)^{\prime} \cap\left(N^{\prime} \cap M_{2}\right)}\left(e_{2}\right) \in \mathbb{C} I$.

Corollary 5.2. There exist only finitely many isomorphism classes of standard commuting squares $\mathfrak{C}$, of fixed dimension $\operatorname{dim} P_{0}$, arising from depth 2 subfactors $N \subset M$ with $E_{\left(N^{\prime} \cap M_{1}\right)^{\prime} \cap\left(N^{\prime} \cap M_{2}\right)}\left(e_{2}\right) \in \mathbb{C} I$.

Remark 5.3. In a depth 2 subfactor, the following is a basic construction: $N^{\prime} \cap M \subset$ $N^{\prime} \cap M_{1} \subset N^{\prime} \cap M_{2}$. For any $A \subset B \stackrel{e}{\subset} C$, a basic construction of finite dimensional $*$-algebras, we can state the previous condition: $E_{B^{\prime} \cap C}(e) \in \mathbb{C} I$. This does NOT always hold true. For instance, it fails for $A=M_{k} \oplus M_{l}$ with $k \neq l$ and $B=M_{n}$, $n=k+l$. We will see however that it holds true if $A \subset B$ has an ONB closed under taking adjoints, and in particular if $A=N^{\prime} \cap M$ is a factor.

Proposition 5.4. With the previous notation, if $N^{\prime} \cap M \subset N^{\prime} \cap M_{1}$ admits an orthonormal basis closed under taking adjoints, then $\mathfrak{C}$ is LRC.

Proof. Let $\left\{a_{i}\right\}_{1 \leq i \leq n}$ be such an orthonormal basis. Then the $a_{i}$ satisfy $\sum a_{i} e_{2} a_{i}^{*}=$ $\sum a_{i}^{*} e_{2} a_{i}=1, \sum a_{i} a_{i}^{*}=\lambda^{-1}$. For $a^{\prime} \in\left(N^{\prime} \cap M_{1}\right)^{\prime} \cap\left(N^{\prime} \cap M_{2}\right)$ we have

$$
\tau\left(e_{2} a^{\prime}\right)=\lambda \tau\left(e_{2} a^{\prime} \sum a_{i} a_{i}^{*}\right)=\lambda \tau\left(\left(\sum a_{i}^{*} e_{2} a_{i}\right) a^{\prime}\right)=\lambda \tau\left(a^{\prime}\right) .
$$

This shows that $E_{\left(N^{\prime} \cap M_{1}\right)^{\prime} \cap\left(N^{\prime} \cap M_{2}\right)}\left(e_{2}\right) \in \lambda I$, which ends the proof.

Corollary 5.5. If $N \subset M$ is a depth 2 subfactor with $N^{\prime} \cap M$ a factor (of type $\left.\mathrm{I}_{\mathrm{n}}\right)$, then the associated depth 2 commuting square is LRC.

Proof. $B=N^{\prime} \cap M_{1}$ must be a tensor product $B=A \otimes S$, where $A$ is the factor $N^{\prime} \cap M$ and $S$ is some *-subalgebra of $B$. Any ONB of $S$ which is closed under taking adjoints is also an ONB for $A \subset B$, closed under taking adjoints. 
In particular, the LRC condition holds if the first relative commutant is trivial, i.e. $N^{\prime} \cap M=\mathbb{C} I$. By a result of $[\mathrm{Szy}$, such commuting squares correspond precisely to the finite dimensional Hopf $C^{*}$-algebras. We thus obtain a new proof of the following theorem of D. Stefan:

Corollary 5.6. For every $N \geq 1$ there exist only finitely many $N$-dimensional Hopf *-algebras.

A somewhat more general class of LRC commuting squares arises from depth 2 subfactors $N=I_{k} \otimes N \subset M_{k}(\mathbb{C}) \otimes M$, where $N \subset M$ is a Hopf algebra cross product subfactor and $I_{k}$ denotes the identity matrix of $M_{k}(\mathbb{C}), k \geq 2$.

\section{Aknowledgements}

The author would like to thank Dietmar Bisch and Sorin Popa for many useful conversations in the past regarding the topic of this paper.

\section{REFERENCES}

[Chr] E.Christensen, Subalgebras of a finite algebra, Mathematische Annalen 243, 17-29 (1979). MR543091 (80i:46051)

[EtNiOs] P. Etingof, D. Nikshych, and V. Ostrik, On fusion categories, Annals of Math. (2) 162 (2005), no. 2, 581-642. MR2183279 (2006m:16051)

$[\mathrm{H}] \quad$ U.Haagerup, Orthogonal maximal abelian $*$-subalgebras of the $n \times n$ matrices and cyclic $n$-roots, Operator Algebras and Quantum Field Theory (ed. S.Doplicher et al.), International Press (1997), 296-322. MR 1491124 (98k:46087)

[Jon] V.F.R.Jones, Index for subfactors, Invent. Math $\mathbf{7 2}$ (1983), 1-25. MR696688 (84d:46097)

[JS] V.F.R.Jones and V.S.Sunder, Introduction to subfactors, London Math. Soc. Lecture Notes Series 234, Cambridge University Press, 1997. MR1473221 (98h:46067)

[Ni] R. Nicoara, A finiteness result for commuting squares of matrix algebras, J. Operator Theory 55 (2006), 295-310. MR2242852 (2007d:46055)

[Oc] A. Ocneanu. Quantized groups, string algebras and Galois theory for von Neumann algebras. Op. Alg. and Apl. London Math. Soc. Lect. Notes Series, 136 (1988), 119172. MR996454 (91k:46068)

[Po1] S.Popa, Classification of subfactors: the reduction to commuting squares, Invent. Math., 101(1990),19-43. MR.1055708 (91h:46109)

[Po2] S.Popa, Classification of amenable subfactors of type II, Acta Mathematica, 172, 163255 (1994). MR 1278111 (95f:46105)

[Po3] S.Popa, Othogonal pairs of *-subalgebras in finite von Neumann algebras, J. Operator Theory 9, (1983), 253-268. MR703810 (84h:46077)

[Po4] S. Popa, An axiomatization of the lattice of higher relative commutants, Invent. Math. 120 (1995), 427-445. MR.1334479 (96g:46051)

[Szy] W. Szymanski, Finite index subfactors and Hopf algebra crossed products, Proc. Amer. Math. Soc., 120(1994),519-528. MR1186139(94d:46061)

Department of Mathematics, University of Tennessee, Knoxville, Tennessee 37996 0612 - And - Institute of Mathematics of the Romanian ACAdemy, 21 Calea Grivitei Street, 010702 Bucharest, Romania 